NASA Technical Memorandum 100273

AIAA-88-0450

\title{
Mass Transport Phenomena Between Bubbles and Dissolved Gases in Liquids Under Reduced Gravity Conditions
}

Kenneth J. DeWitt

University of Toledo

Toledo, Ohio

Jonathan L. Brockwell

Union Carbide Corporation

South Charleston, West Virginia

and

Chain-Nan Yung, An-Ti Chai, John B. McQuillen, Raymond G. Sotos, Eric S. Neumann

Lewis Research Center

Cleveland, Ohio

Prepared for the

26th Aerospace Sciences Meeting

sponsored by the American Institute of Aeronautics and Astronautics Reno, Nevada, January 11-14, 1988

\section{NMSก}

(MASA-TH-100273) DASS TEANSECBI FHENOHENA

$888-18672$

EETEREN EOBBLES AD DISSCIYED GASES IN

LICOIDS ONDER FELUCED GFAVITY CCADITIONS

(AASA) $10 \mathrm{p}$

CSCL 07D

$\begin{array}{ll}\text { Onclas } \\ 63 / 25 & 0128793\end{array}$ 


\title{
MASS TRANSPORT PHENOMENA BETWEEN BUBBLES AND DISSOLVED GASES IN LIQUIDS UNDER REDUCED GRAVITY CONDITIONS
}

\author{
Kenneth J. DeWitt* \\ University of Toledo, Toledo, Ohio \\ Jonathan L. Brockwell \\ Union Carbide Corporation, South Charleston, West Virginia \\ Chain-Nan Yung, \\ An-Ti Chai, \\ John B. McQuillen, \\ Raymond G. Sotos, \\ Eric S. Neumann \\ NASA Lewis Research Center, Cleveland, Ohio
}

\begin{abstract}
This paper will describe the experimental and analytical work that has been done to establish justification and feasibility for a shuttle mid-deck experiment involving mass transfer between a gas bubble and a liquid. The experiment involves the observation and measurement of the dissolution of an isolated, immobile gas bubble of specified size and composition in a thermostatted solvent liquid of known concentration in the reduced gravity environment of earth orbit. Methods to generate and deploy the bubble have been successful both in normal gravity using mutually buoyant fluids and under reduced gravity conditions in the NASA Lear Jet. Initialization of the experiment with a bubble of a prescribed size and composition in a liquid of known concentration has been accomplished using the concept of unstable equilibrium. Subsequent bubble dissolution or growth is obtained by a step increase or decrease in the liquid pressure. A numerical model has been developed which simulates the bubble dynamics and can be used to determine molecular parameters by comparison with the experimental data. The primary objective of the experiment is the elimination of convective effects that occur in normal gravity. The results will yield information on transport under conditions of pure diffusion.
\end{abstract}

\section{Introduction}

Whenever a gas and a liquid are in contact, mass transfer across the gas-liquid interface will take place as an equilibrium is approached between the partial pressure of a species in the gas phase and the concentration of the dissolved species in the liquid. In the case of a bubble in a liquid, the bubble may either grow in size, diminish in size, or remain in unstable equilibrium, depending upon the concentration of the transferring species in the surrounding liquid, the system pressure and temperature, and the initial bubble radius. The overall rate of transfer of a gas between a bubble and a surrounding liquid is a function of the coupled effects of molecular diffusion, surface phenomena and hydrodynamics.

It is desirable to uncouple these phenomena so that an increased understanding of the roles molecular diffusion and interfacial effects play in the mass transfer process involving single bubbles can be obtained. This is difficult to do in normal gravity, as a gas bubble will rise through a liquid due to the difference in phase densities (buoyancy), which couples the diffusional mass transfer with the hydrodynamics of buoyant bubble motion. The buoyant motion affects the mass transfer process in a complicated way by disrupting the distribution of dissolved gas in the liquid and by distorting the bubble shape from total sphericity. In addition, liquid density variations due to a non-uniform distribution of dissolved gas can produce free convection velocities which will influence the overall process.

In order to observe bubble behavior in the absence of buoyant translation, it is necessary to immobilize the bubble. This can be accomplished by restraining the bubble by attachment to a solid surface. This prevents the buoyant rise of the bubble so that the distribution of dissolved gas in the liquid is not disrupted by large scale bubble translation. However, the presence of the attaching surface can hinder the mass transfer and the distortion of the bubble shape from total sphericity due to buoyant forces can alter the radial symmetry of the dissolution process. There is also some small translation of the bubble relative to its point of attachment as the bubble grows or shrinks. In addition, free convection can be significant for some gas-liquid systems. Therefore, immobilization of a gas bubble does not eliminate the influence of gravity on the dissolution process, but changes it; perhaps reducing it under some conditions.

A free-fall or in-space environment provides a setting for experimentation from which buoyant bubble translation, obstruction by solid surfaces and free convection are absent. Careful observation of bubble growth and dissolution in such an environment could more clearly demonstrate the enhancement of the mass transfer process which results from the coupling of diffusion and hydrodynamic effects in a translating bubble. 


\section{Background}

The mathematical description of the growth or dissolution of a stationary, spherically symmetric gas bubble in a liquid pool by diffusional mass transfer is a "Stefan-like" problem. It is a boundary value problem where boundary conditions are specified at a phase transition front which moves with time. The movement of the front is not specified beforehand, but is dependent upon the rate of mass transfer. Therefore, the changing solute concentration in the liquid and the movement of the phase transition front with time must be solved for simultaneously. In addition, the transfer of material between phases of different densities, as in the case of gas-in-liquid systems, produces a radial convective motion in the surrounding liquid which modifies the concentration field and, consequently, the motion of the front. The inclusion of the radial convective term in the conservation of species equation makes the resulting partial differential equation nonlinear.

Analytical and numerical efforts to solve these equations have been made. Scriven ${ }^{1}$ and $\mathrm{TaO}^{2,3}$ have developed exact solutions of these equations. Scriven's solution was based on the use of a similarity transform and was valid only for bubble growth from an initial zero radius. Tao's results apply for both bubble growth or shrinkage from a non-zero initial size. However, Tao neglected the convective velocity in the diffusion equation, which makes his solution valid only for the situation where the concentration gradient in the liquid is small. In this circumstance, the rate of change of the concentration boundary layer thickness is fast compared to the rate of the movement of the bubble interface ${ }^{8,16}$.

In the attempt to obtain approximate solutions, there have been numerous approaches, e.g., integral methods $4,5,17$, a perturbation method ${ }^{6}$, quasi-stationary methods $7,8,19$, thin shell approximations 9,18 , and a short time asymptotic expansion technique ${ }^{10,11}$. The areas of application of these approximate methods are confined within small operating ranges. For instance, the quasistationary solution is obtained by ignoring the convective transport term in the diffusion equation, which is the same as Tao's approach. The thin shell solution applies to the situation where the mass diffusion length is small in comparison with the bubble radius. The motion of the bubble interface is larger than the variation of the thickness of the concentration layer. The solution can thus be regarded valid for a large concentration driving force ${ }^{15}$. The short time expansion method ${ }^{10,11}$ is based on the fact that in the limit as time approaches zero, a similarity solution may be found for the concentration field. A series solution is thus developed by expanding the similarity solution in time to higher orders. The results of a third order expansion compared favorably with the finite difference solution for the small to moderate time span ${ }^{10}$. Since this technique is short time asymptotic in nature, it is not expected to provide an accurate solution for large times in bubble growth problems.

The utility of finite difference methods was examined by several authors ${ }^{12-15}$. This method allows physical parameters and flow variables, e.g., surface tension and gas expansion in the bubble, to be easily implmented into the mathematical model and boundary conditions. The present paper discusses a finite difference method to solve the system equations without any of the restrictive limitations discussed above.
All of the existing analyses of mass transfer between an isolated immobile bubble and a liquid treat the process as though it were occurring in the absence of gravity. There are articles in the literature which report research into the effects of buoyant free convection on heat and mass transfer processes ${ }^{20-22}$. However, none have dealt with the influence of buoyant free convection on the bubble growth or dissolution process where a moving boundary between phases of different densities produces convection in what otherwise would be still fluid. Experiments in space would provide reduced gravity data which could be compared to normal gravity data to assess the importance of buoyancy forces in the mass transfer process in normal gravity.

Several papers are available in the literature which describe experiments in which the disappearance with time of a bubble immobilized in normal gravity was observed ${ }^{23-29}$. The primary aim of the experimentation was to determine diffusion coefficients for the various gas-liquid systems studied. Buoyant bubble translation was arrested by attaching the bubble to a finely drawn fiber or by trapping it against a flat plate. A predicted radius-time relationship derived from a model which ignored the effects of gravity entirely was then applied to the data and the diffusion coefficient adjusted to obtain the best agreement.

Although immobilization of a bubble by attachment to a solid surface eliminates large scale bubble translation, the attaching surface acts as a barrier to diffusion and liquid convection. Furthermore, immobilization of a gas bubble does not eliminate the influence of gravity, but alters its effect on the bubble behavior from that observed for freely rising bubbles. Because a hydrostatic pressure gradient exists across the bubble, in instances where surface tension effects are small or negligible, the attached bubble assumes a nonspherical cross-section. This deviation of the bubble from total sphericity destroys the radial symmetry of both the liquid convection and solute concentration fields. In addition, there remains some small translation of the bubble center of mass relative to its point of attachment as the bubble shrinks. Kreiger et al. ${ }^{23}$ stated that the small liquid velocities produced as the surface of the bubble moves inward and its center moves downward may have been the principal cause for the deviations in their derived diffusion coefficients. Also, buoyant free convection can occur in the liquid as the bubble dissolves due to concentration dependent density gradients. In some cases free convection can significantly alter the solute distribution in the liquid and thereby influence the bubble dissolution process.

Both Houghton et al. ${ }^{26}$ and Wise and Houghton ${ }^{27,28}$ suggest concentration induced free convection as a possible contributor to the variability in their experimentally derived diffusion coefficients. Pfeiffer and Krieger ${ }^{29}$ stated that the elimination of buoyant free convection due to concentration and thermal gradients was a critical experimental problem. Their results indicated a significant correlation between the derived diffusion coefficient and the size of the bubble used to make the raw measurements. The authors discussed the role of bubble size on free convection in terms of the Grashof number. They concluded from an inspection of the magnitudes of the Grashof numbers appropriate to the systems studied that the use of small bubbles probably reduced the effect of free convection on the results. 
The accuracy of a diffusion coefficient derived from measurements made on an immobilized bubble dissolving in normal gravity would depend greatly on the extent to which the influences of attachment and gravity can be quantified. These effects at present have been characterized only on a largely qualitative basis. An experimental environment which provided true isolation of a stationary bubble in the absence of gravity would eliminate the need to deal with these effects.

The dissolution of a single gas bubble in a liquid under reduced gravity conditions has not yet been studied. This environment would provide a means of immobilizing the bubble in true isolation and eliminating buoyant free convection. Such an environment much more closely fulfills the qualifying assumptions made in the development of existing bubble dissolution models which ignore gravity altogether. Diffusion coefficients derived from fitting predicted radius-time behavior from an appropriate model to reduced gravity measurements should be of a higher order of accuracy than those obtained under normal gravity conditions.

\section{Experimental and Analytical Work}

Preliminary bubble dissolution experiments ${ }^{30,31}$ conducted both in the NASA Lewis $2.2 \mathrm{sec}$. droptower and in normal gravity using the $\mathrm{SO}_{2}$-toluene system were not completely successful in their objective. The choice of this extremely soluble system was based on the expected dissolution response in the $2.2 \mathrm{sec}$. of free-fall time available in the tower. However, the method of gas injection and the lack of bubble interface stability experienced due to the extreme solubility of $\mathrm{SO}_{2}$ in toluene had the effects of changing the problem from that of bubble dissolution from a known initial condition to one of bubble formation and subsequent slow dissolution in a liquid of unknown initial solute concentration.

The method of gas injection used in the droptower tests was by means of a spring-actuated hypodermic syringe. In 12 of the 18 experiments in which bubbles were produced, the resulting bubble formation rate was either too slow so that the bubble remained attached to the syringe needle tip due to surface tension effects, or the formation rate was too high, such that after detaching from the needle tip, the bubble quickly crossed and disappeared from the photographic field of view due to inertia effects. Bubble oscillation, swarming, and coalesence were other consequences of the method of injection, although the system solubility also contributed to the observed phenomena. In the 6 droptower experiments in which acceptable $\mathrm{SO}_{2}$ bubbles were produced, the resulting bubble size was such that only about $50 \%$ of the injected $\mathrm{SO}_{2}$ appeared in the resulting bubble; the remainder of the $\mathrm{SO}_{2}$ had been immediately absorbed by the toluene, thus partially saturating the toluene so that a continuous gasliquid interface could be stabilized. The presence of a region of partially saturated toluene immediately around a newly formed and detached $\mathrm{SO}_{2}$ bubble caused a more abrupt transition between the early absorption controlled rapid dissolution and the later diffusion controlled phase of the dissolution process, and produced a lower dissolution rate over the latter portion of the free-fall period than was anticipated had the bubble been immersed in $\mathrm{SO}_{2}$-free toluene from the beginning. Figures 1 and 2 illustrate the high, then slow, rate of bubble dissolution experienced in cases 141 and $14-2$ in the droptower tests. Since concentration

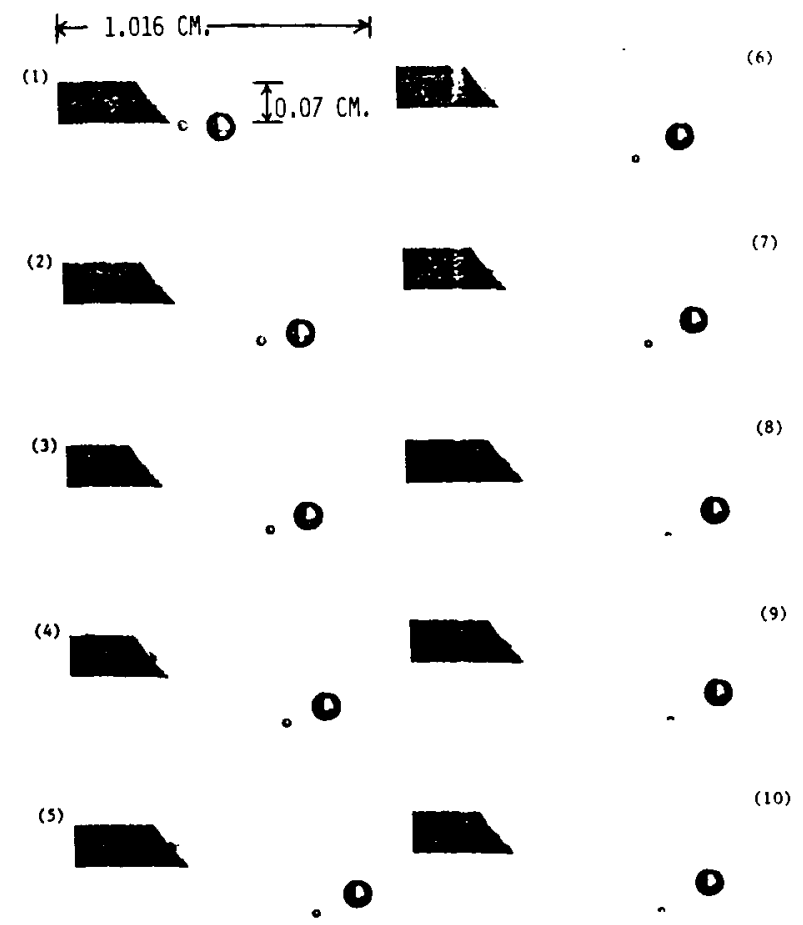

Fig. 1. Sequence of Bubble Dissolution. Original Bubble Diameters $0.053 \mathrm{~cm}$. and $0.020 \mathrm{~cm}$. Final Diameters $0.048 \mathrm{~cm}$. and $0.015 \mathrm{~cm}$. Elapsed Time Between Frames $0.22 \mathrm{Sec}$.

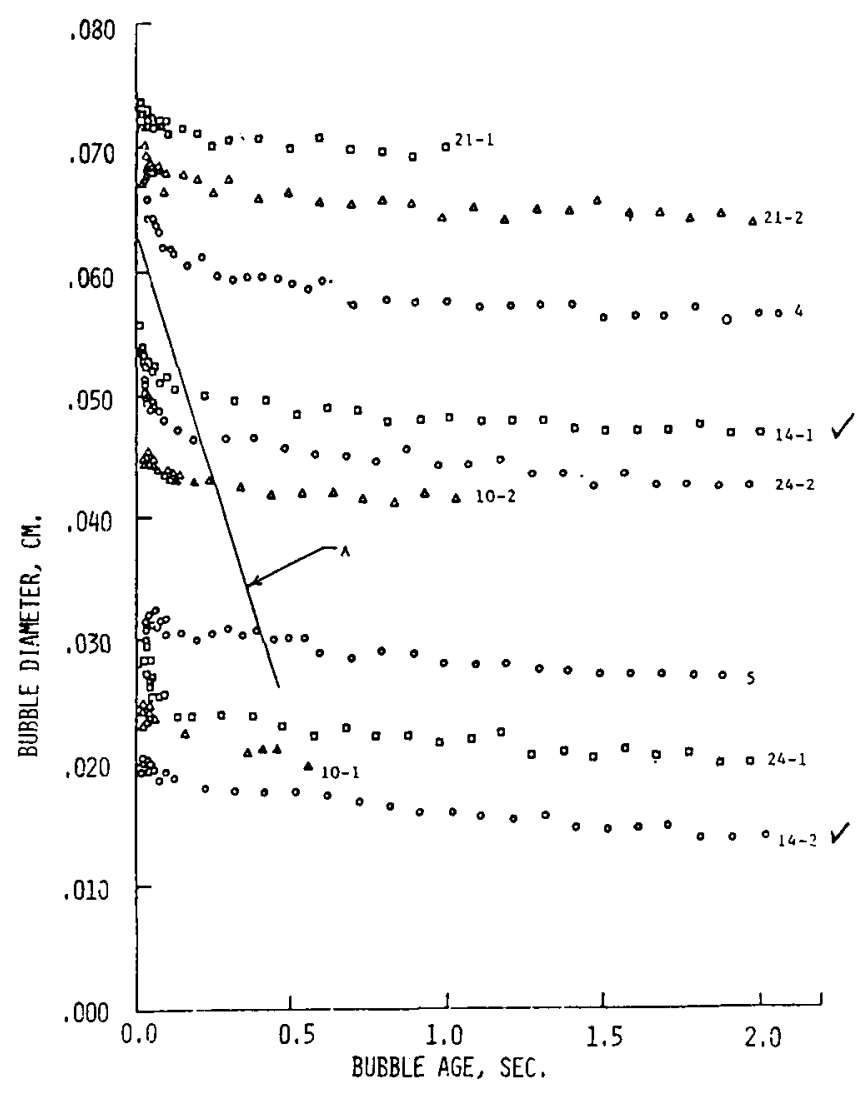

Fig. 2. Bubble Diameter Versus Bubble Age from Droptower 
measurements in the surrounding liquid could not be obtained in these experiments, the subsequent bubble dissolution data was difficult to interpret due to the unknown initial concentration distribution in the liquid. Further feasibility work was decided to be necessary on the subjects of bubble initialization and bubble injection.

\section{Bubble Initialization}

Bubble initialization, deployment of a bubble of known size in a liquid of uniform composition from which bubble growth or dissolution proceeds, can be achieved by means of the critical radius phenomenon. At critical radius conditions, a bubble is in unstable equilibrium with the surrounding liquid and is neither growing nor dissolving. To initiate bubble growth or dissolution, the system pressure is changed from its value at critical radius conditions by an amount large enough to produce measurable change, but not so large as to disrupt the bubble.

Ward et al ${ }^{32}$ derived an expression for the change in the Helmholtz free energy as a function of the bubble radius in the neighborhood of the critical radius. The conditions of equilibrium and the derived free energy expression show that an equilibrium exists at a critical radius $R_{c}$, but the equilibrium is an unstable one. If a bubble is smaller than the critical radius, it will shrink. A bubble larger than the critical radius will grow. The authors went on to develop an expression for the critical radius for a weak solution of gas dissolved in a liquid. A weak solution is one in which the number of solute molecules is much less than the number of solvent molecules. The critical radius expression for a weak binary system shown in Figure 3 is explicit in system pressures and concentrations. If the pressures and

WEAK SOLUTION - Number of Molecules of Dissolved Solute Number of Molecules of Solvent

CONDITIONS:

$$
\begin{aligned}
& \mu_{i} \text { Bubble }=\mu_{i} \text { Liquid }=\mu_{i} \text { Interface } \\
& \mathrm{P}^{\text {Bubble }} \cdot \mathrm{P}^{\text {Liquid }}=2 \sigma / \mathrm{R}
\end{aligned}
$$

\section{RESULTS:}

$$
\begin{array}{ll}
\mathrm{R}_{\mathrm{c}} & =2 \sigma /\left(\eta \mathrm{P}_{\text {vap }}+\mathrm{P}^{\prime} \mathrm{c}^{\prime} / \mathrm{c}_{\mathrm{o}}-\mathrm{P}^{\prime}\right) \\
\sigma & =\text { Liquid }- \text { Gas Interfacial Tension } \\
\mathrm{P}^{\prime} & =\text { Pressure in Liquid } \\
\mathrm{c}^{\prime} & =\text { Gas Concentration in Liquid } \\
\mathrm{P}_{\mathrm{vap}} & =\text { Vapor Pressure of Pure Solvent } \\
\eta & =\exp \left\{\left[\mathrm{v}_{\mathrm{L}}\left(\mathrm{P}^{\prime}-\mathrm{P}_{\text {vap }}\right)-\mathrm{k} \mathrm{T} \mathrm{c}^{\prime}\right] / \mathrm{kT}\right\} \\
\mathrm{v}_{\mathrm{L}} & =\text { Specific Volume of Pure Solvent } \\
\mathrm{T} & =\text { Temperature } \\
c_{\mathrm{O}} & =\text { Gas Concentration in Liquid Saturated } \\
\mathrm{k} & =\text { with Gas Across a Flat Surface }
\end{array}
$$

Fig. 3. Critical Radius for "Weak" Solution concentrations are known, the critical radius can be computed directly. Development of an explicit critical radius expression was made possible by the existence of chemical potential expressions which are explicit in pressure and concentration.

Tucker and Ward ${ }^{33}$ reported results of an experimental study of oxygen bubbles in water near the critical radius. A sequence of photographs of two bubbles simultaneously present in the liquid show the one slightly larger than critical radius to grow and the other slightly smaller than critical size to dissolve. This demonstrated that the critical radius lay between the initial radii of the two bubbles and that the critical state was indeed unstable. Further tests were conducted with the water-oxygen system to more carefully compare predicted and observed critical radii.

Ward and Tucker $^{34}$ utilized the critical radius phenomenon to control initial conditions in a study of the dissolution of oxygen-nitrogen bubbles in water. The authors state that use of the critical radius phenomenon simultaneously solved three problems associated with bubble evolution studies: first, initial solute concentrations at the interface could be determined; second, concentrations in the liquid were uniform and well defined; and third, the bubble radius at time equals zero could be determined accurately. In their procedure, the desired gas content in the liquid was established by bubbling an oxygen-nitrogen mixture through the water, after which an approximately critically sized bubble was injected into the liquid and caught on a quartz fiber. The pressure was adjusted to hold the bubble at the critical size, until dissolution was begun by applying a small pressure increase to the system. The initial radius was determined by assuming a linear dissolution rate between two photographs taken a short time apart following the pressure increase and extrapolating bubble size to time equals zero.

The expression derived by Ward et al. for the free energy change in the neighborhood of the critical radius is of general applicability. Under appropriate conditions a bubble can exist in unstable equilibrium with a surrounding liquid when the gas is more than slightly soluble in the solvent liquid, i.e., a non-weak system. However, an explicit expression for the critical radius like that for weak systems is not available for non-weak systems. Chemical potential expressions explicit in temperature, pressure and concentrations like those used to develop the weak system critical radius expression are not known for non-weak systems.

Initial estimates of the critical radii for a non-weak system were made using equations (1a) and (1b).

$$
\begin{gathered}
\mathrm{R}_{\mathrm{c}}=2 \sigma /\left(\eta \varphi_{1} \mathrm{x}_{1} \mathrm{P}^{\prime}+\varphi_{2} \mathrm{x}_{2} \mathrm{P}^{\prime}-\mathrm{P}^{\prime}\right) \\
\eta=\exp \left\{\left[\mathrm{v}_{\mathrm{L}}\left(\mathrm{P}^{\prime}-\mathrm{P}_{\text {sat'd }}\right)\right] / \mathrm{k}\right\}
\end{gathered}
$$

The critical radius expression shown is analogous to the expression for weak systems with liquid-phase fugacities, $\varphi_{i}$, replacing pressure terms in the denominator. It can be used to compute the critical radius if appropriate physical properties and liquidphase fugacities are available. Measured pressuretemperature-composition data for the $\mathrm{CQ}_{2}$-toluene system were published by $\mathrm{Ng}$ and Robinson ${ }^{3}$. These data were used to determine parameters in the Starling modification of the Benedict-Webb-Rubin equation of state. Liquid-phase fugacity coefficients at selected temperatures, pressures and solution compositions were 
calculated using the equation of state. In view of the uncomplicated equilibrium conditions, an iterative scheme to compute the critical radius (or alternatively, the pressure at criticality) should be straightforward. A computer-based iterative computation scheme has been developed, and calculations were made of critical radius conditions for the $\mathrm{CO}_{2}$-toluene system.

An apparatus to study the critical radius phenomenon in normal gravity was constructed. The cell used is shown in Figure 4. With this apparatus it was possible to inject a bubble of gas into the solvent solution, restrain the bubble beneath a group of parallel fibers, adjust the pressure to achieve criticality and observe growth or shrinkage of the bubble as the system pressure was changed. A number of trials with $\mathrm{CO}_{2}-$ toluene solutions have been run using the normal gravity apparatus in an attempt to demonstrate the critical radius phenomenon with this system. Data from two trials are summarized in Table 1 and in Figures 5 and 6. In the first trial, shown in Figure 5, a bubble was injected and held at conditions such that its size did not change over a ten-minute period. A pressure decrease of 0.25 psia caused the bubble to grow more than twice its original radius over the ensuing five-minute period. In the second trial, shown in Figure 6, a bubble was held at critical radius conditions for a four-minute period, at which time a pressure increase of 0.15 psia caused the bubble to dissolve to less than one-half of its original radius over the next five-minute period. These results demonstrate the feasibility of using the critical radius phenomenon for bubble initialization with non-weak gas-liquid systems.

Table 1. Bubble Initialization

\begin{tabular}{|c|c|c|c|}
\hline \multicolumn{4}{|c|}{$\begin{array}{l}\text { Growing Bubble } \\
\text { Date: } 9-24-87 \\
\text { Saturation Pressure }=8.000 \text { psia }\end{array}$} \\
\hline $\begin{array}{l}\text { Time } \\
(\min )\end{array}$ & $\begin{array}{l}\text { Pressure } \\
\text { (psia) }\end{array}$ & $\begin{array}{l}\text { Temp. } \\
\left({ }^{\circ} \mathrm{C}\right)\end{array}$ & $\begin{array}{r}\text { Radiu } \\
(\mathrm{mm})\end{array}$ \\
\hline $\begin{array}{l}2: 19 \\
2: 21 \\
2: 23 \\
2: 25 \\
2: 27 \\
2: 28 \\
2: 29\end{array}$ & $\begin{array}{l}7.950 \\
7.950 \\
7.950 \\
7.950 \\
7.950 \\
7.950 \\
7.950\end{array}$ & $\begin{array}{l}18.0 \\
18.0 \\
17.5 \\
17.5 \\
17.5 \\
17.5 \\
17.5\end{array}$ & $\begin{array}{l}0.643 \\
0.643 \\
0.643 \\
0.643 \\
0.643 \\
0.643 \\
0.643\end{array}$ \\
\hline \multicolumn{4}{|c|}{ Pressure Decrease of 0.25 psia } \\
\hline $\begin{array}{l}2: 30 \\
2: 32 \\
2: 33 \\
2: 34\end{array}$ & $\begin{array}{l}7.700 \\
7.700 \\
7.700 \\
7.700\end{array}$ & $\begin{array}{l}17.8 \\
17.8 \\
17.8 \\
17.8\end{array}$ & $\begin{array}{l}0.878 \\
1.146 \\
1.353 \\
1.484\end{array}$ \\
\hline
\end{tabular}

Dissolving Bubble

Date: 8-18-87

Saturation Pressure $=5.800$ psia

$\begin{array}{llll}11: 50 & 6.026 & 29.5 & 1.320 \\ 11: 51 & 6.026 & 29.4 & 1.340 \\ 11: 52 & 6.027 & 29.4 & 1.350 \\ 11: 53 & 6.027 & 29.3 & 1.350 \\ 11: 54 & 6.027 & 29.4 & 1.350\end{array}$

Pressure Increase of 0.156 psia

$\begin{array}{llll}11: 55 & 6.183 & 29.2 & 1.290 \\ 11: 56 & 6.183 & 29.2 & 1.130 \\ 11: 57 & 6.183 & 29.3 & 1.070 \\ 11: 58 & 6.183 & 29.3 & 0.860 \\ 11: 59 & 6.183 & 29.3 & 0.670\end{array}$

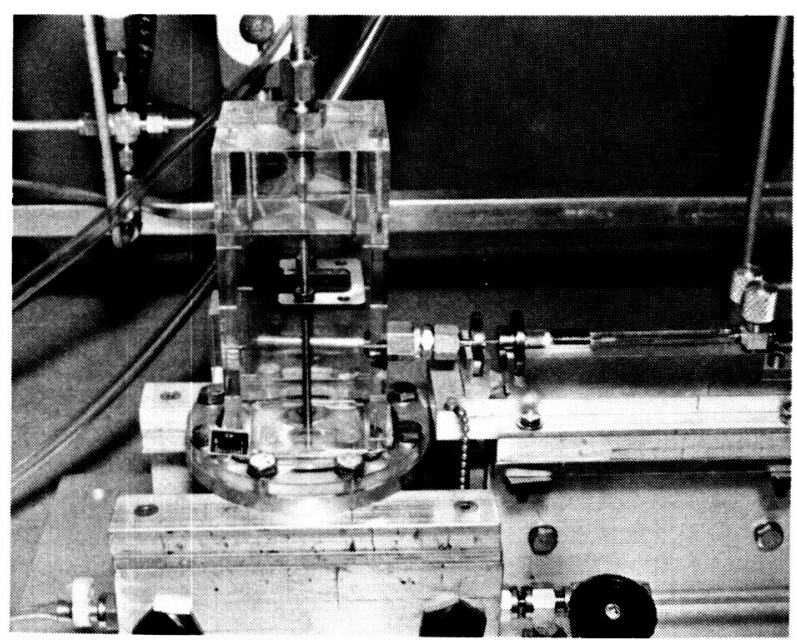

Fig. 4. Normal Gravity Cell with Fibers

$$
t=8 \mathrm{~min} .
$$

2:27 p.m.

$t=6 \mathrm{~min}$
2:25 p.m.

$t=15 \mathrm{~min}$
2:34 p.m.

$t=4 \min$

$2: 23$ p.m.

$t=13 \mathrm{~min}$
2:32 p.m.

$\rightarrow \mathrm{t}=2 \mathrm{~min}$.

2:21 p.m.

$\rightarrow \quad t=11 \mathrm{~min}$

$\mathrm{P}=7.70$ psia
$\mathrm{t}=0 \mathrm{~min}$. 2:19 p.m. $\mathrm{P}=7.95 \mathrm{psia}$
(- $t=10 \mathrm{~min}$.

Fig. 5. Growing Bubble

Saturation Pressure $=8.0$ psia

Temperature $=18^{\circ} \mathrm{C}$

\section{Bubble Injection and Deployment}

Bubble injection and deployment was studied using two types of injection devices. The first type of injector was a double barrel device where the inner barrel containing the gas slides within the outer barrel. Both barrels possess circumferential holes with o-ring seals 


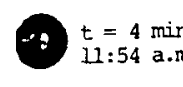

$t=9 \mathrm{~min}$.
$11: 59 \mathrm{a} . \mathrm{m}$.

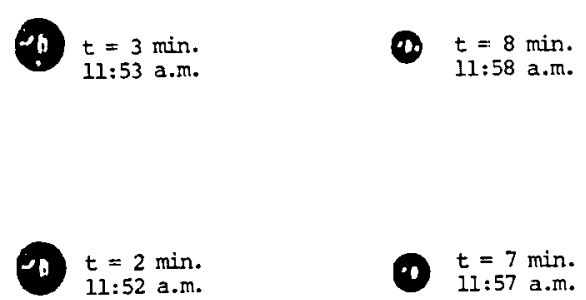

(4) $\begin{aligned} & t=1 \mathrm{~min} . \\ & 11: 51 \mathrm{a} \cdot \mathrm{m} .\end{aligned}$

$\begin{array}{ll}t=0 \mathrm{~min} . & t \\ 11: 50 \mathrm{a} . \mathrm{m} . & t=5 \mathrm{~min} . \\ p=6.026 \mathrm{psia} & 11: 55 \mathrm{a} . \mathrm{m} . \\ P=6.183 \mathrm{psia}\end{array}$

Fig. 6. Dissolving Bubble

Saturation Pressure $=5.8 \mathrm{psia}$

Temperature $=29.5^{\circ} \mathrm{C}$

surrounding the hole in the inner barrel. When the holes line up, gas is allowed to escape. Gas pressure and resulting inertia control the bubble size and deployment. This injector was tested on the NASA Lear Jet and produced results on injection and deployment which satisfy this feasibility issue. The second injector was a concentric tube, positive displacement device whereby the gas is injected through the inner tube and bubble growth occurs at the end of the tube. The bubble is subsequently displaced from the end of the inner tube by a push from liquid coming through the outer tube. This liquid push overcomes surface tension and frictional forces and deploys the bubble. This injector was tested under normal gravity conditions using mutually buoyant fluids. Again, acceptable feasibility results were obtained.

\section{Numerical Simulation}

The equations describing the growth or dissolution of a spherical gas bubble in a liquid under reduced gravity conditions were solved using a finite difference procedure ${ }^{36}$. The bubble was initially in unstable equilibrium, the gas interior to the bubble assumed uniform and ideal, and the liquid assumed to be incompressible. The equation of motion and the species continuity equations in the liquid phase were solved with the appropriate thermodynamic and mechanical boundary conditions. Gas expansion inside the bubble, surface tension, liquid viscosity and the presence of solvent vapor in the bubble were included in the model. Upwind differencing was incorporated into the finite difference procedure in order to avoid solution oscillation. Results computed from the model compared very well with the experimental dissolultion data of Ward et al. ${ }^{17}$ for oxygen bubbles in water, Krieger et al. ${ }^{23}$ for the same system, and with Ward and Tucker $^{23}$ for nitrogen-oxygen bubbles in water. This simulation will be used for comparison with future reduced gravity bubble dissolution or growth data, and also with normal gravity data currently being obtained for a selected set of gas-liquid systems.

\section{Effect of Acceleration Forces}

In any shuttle experiment, there will still be acceleration forces or "g-jitter" that could disrupt the phenomena trying to be observed. An analysis was made to quantify any effects that g-jitter could have on the present mass transfer experiment. The two issues of concern were: (1) whether mass transfer by diffusion will dominate convective mass transfer caused by the acceleration forces; and (2) whether natural convection mass transfer will occur. The first issue was answered by the calculation of a Peclet Number for mass transfer, (D V/D $\mathrm{D}_{\mathrm{AB}}$ ), where $\mathrm{D}$ is the bubble diameter, $\mathrm{V}$ is the bubble velocity caused by g-jitter, and $\mathrm{D}_{\mathrm{AB}}$ is the binary diffusivity. The Peclet Number is a measure of bulk mass transfer divided by diffusion mass transfer. Since this number must be less than 1.0 for diffusion to dominate, a calculation for the $\mathrm{CO}_{2}$-toluene system yielded the information that acceptable results would be obtained using a bubble of $0.2 \mathrm{~mm}$. in diameter at acceleration levels of $10^{-4} \mathrm{~g}$ or lower. This force would impart a velocity to the bubble of $0.03 \mathrm{~cm} . / \mathrm{min}$., which yields a Peclet Number of 0.22 . The second issue required calculation of a Rayleigh Number for these conditions. This calculation yielded a value of 0.5 , which is much less than the critical value needed for free convective mass transfer to be important. In summary, for $g$ levels of $10^{-4}$ or lower, acceptable experimental results will be obtained by the use of bubbles whose diameter is $0.2 \mathrm{~mm}$. or less.

\section{Description of Reduced Gravity Experiment}

Based on the above experimental and theoretical work which confirmed the feasibility of conducting a reduced gravity bubble dissolution experiment in space, further work was undertaken to specify the objectives of the experiment, the minimum set of experiments, and the major parts of the required apparatus.

\section{Experimental and Theoretical Objectives}

The long duration, weightless environment of earth orbit provides a unique setting in which to observe bubble-liquid mass transfer. From this environment free convection, buoyancy and the interfering influences of physical restraints used to arrest the buoyant rise of a bubble in normal gravity are absent. The object of the proposed experiment is to observe the dissolution of isolated, immobile gas bubbles of specified size and composition in a solvent liquid of known concentration in the reduced gravity environment of earth orbit. Bubble size measurements taken from a time-sequence of photographs will provide a quantitative record of the radius-time behavior of the dissolving bubble. The theoretical objectives are to use the data to (1) assess the influence of gravity as manifested by convective effects (buoyancy) on the mass transfer process by comparison with normal gravity results; (2) determine 
criteria specifying the conditions under which buoyant free convective effects are significant in normal gravity; (3) screen the available zero-gravity bubble dissolution models by comparing the general features of predicted dissolution curves with the radius-time behavior observed; and to (4) determine diffusion coefficients for the gas-liquid systems studied by adjusting parameters in the selected models to best fit the observed results.

\section{Minimum Set of Experiments}

The minimum set of experiments to be conducted in earth orbit consists of a series of bubble injections with the $\mathrm{CO}_{2}$-toluene system. $A$ total of six successful experiments is required to observe bubble dissolution at two temperatures and three solution compositions. The initial bubble size is constant, and the system pressure is fixed by the temperature-composition-bubble size combination. Proposed temperatures and compositions for experiments using the $\mathrm{CO}_{2}$-toluene system are: Temperatures: 20 and $30^{\circ} \mathrm{C}$;

$\mathrm{CO}_{2}$ Concentrations: Approximately 20,50 and 80 mole percent of the equilibrium solubility at $20^{\circ} \mathrm{C}$ and 1 atm. pressure.

An expanded set of experiments would include observations with at least one other gas-liquid system and possibly additional trials using the $\mathrm{CO}_{2}$ toluene system. Other gas-liquid systems being considered are $\mathrm{N}_{2}$-toluene, $\mathrm{CO}_{2}$-heptane, $\mathrm{N}_{2}$-ethanol and $\mathrm{CO}_{2}$ ethanol. Experiments with one or more of these systems would duplicate the minimum set of trials specified for the CO2-toluene system.

\section{Required Apparatus}

A simplified drawing of the required apparatus is shown in Figure 7. There are several major components required. These are (1) the test cell and associated items; (2) the pressure regulation system; (3) the imaging system; (4) the bubble injection system; (5) the concentration measuring system; (6) the solvent preparation system; and (7) the data acquisition and control system. Detailed specifications of sizes, conditions, measurement accuracy and crew involvement for each of these components is currently underway.

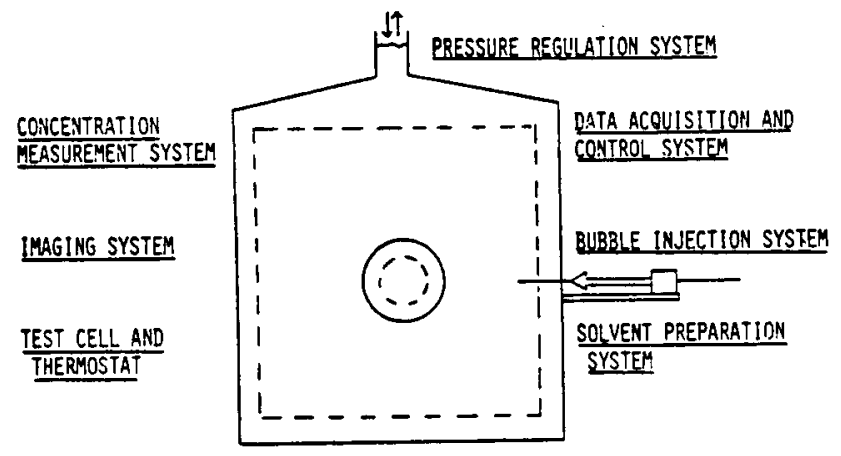

Fig. 7. General Features of Apparatus

\section{Summary}

The potential applications to the science of gasliquid mass transfer to be derived from this study are that the data (radius-time relationships, diffusion coefficients) would provide a highly reliable information base for the development of a general theory of diffusion of gases in liquids. In addition, the data constitutes the initial information needed for future studies of chemical absorption and multiple bubble selective absorption systems. The results should yield information on molecular transport under conditions of pure diffusion and will have applications to many problems in physical chemistry and technology.

\section{Acknowledgement}

This work is being supported by the NASA Lewis Research Center, Cleveland, Ohio. Special thanks are due to Mr. Jack Salzman of NASA Lewis.

\section{References}

1. Scriven, L. E., Chem. Eng. Sci. 10, 1, 1959.

2. Tao, L. N., J. Chem. Phys. $69,4189,1978$.

3. Tao, L. N., J. Chem. Phys. 71, 3455, 1979.

4. Theofanous, T. G., H. S. Isbin and H. K. Fauske, AICHE J. 16, 688, 1970.

5. Rosner, D. E. and M. Epstein, Chem. Eng. Sci. 27, 69, 1972.

6. Duda, J. A. and J. S. Vrentas, AICHE J. 15, 351, 1969.

7. Epstein, P. S. and M. S. Plesset, J. Chem. Phys. $18,1505,1950$.

8. Weinberg, M. C., P. I. K. Onorato and D. R. Uhlmann, J. Amer. Cerm. Soc. 63, 175, 1980.

9. Barlow, E. J. and W. E. Langlois, IBM J., 329, July, 1972.

10. Subramanian, R. S. and M. C. Weinberg, AICHE J. 27, 739, 1981.

11. Shankar, N., T. J. Wiltshire and R. S. Subramanian, Chem. Eng. Comm. 27, 263, 1984.

12. Cable, M. and D. J. Evans, J. Appl. Phys. 38, 2899, 1967.

13. Readey, D. W. and A. R. Cooper, Jr., Chem. Eng. Sci. $21,917,1966$.

14. Szekely, J. and G. P. Martins, Chem. Eng. Sci. 26, 147, 1971.

15. Duda, J. A. and J. S. Vrentas, J. Heat Trans. 14, 395, 1971.

16. Subramanian, R. S. and M. C. Weinberg, J. Chem. Phys. 72, 6811, 1980.

17. Ward, C. A., M. Rizk and A. S. Tucker, J. Chem. Phys. 76, 5606, 1982. 
18. Plesset, M. S. and S. A. Zwick, J. Appl. Phys. 25, 393, 1954.

19. Hsieh, D. Y., J. Basic Eng., 991, December, 1965.

20. Hellums, J. D. and S. W. Churchill, Chem. Eng. Prog. Symposium Series 57, 75, 1960.

21. Saville, D. A. and S. W. Churchill, J. Fluid Mech. 29, 391, 1967.

22. Mathers, W. G., A. V. Madden, Jr. and E. L. Diret, Ind. \& Eng. Chem. 49, 961, 1957.

23. Krieger, I. M., G. W. Mullholland and C. S. Dickey, J. Phys. Chem. 71, 1123, 1967.

24. Liebermann, L., J. Appl. Phys. 28, 205, 1957.

25. Manley, D. J. M. P., Brit. J. Appl. Phys. 11, 38, 1960 .

26. Houghton, G., P. D. Ritchie and J. A. Thomson, Chem. Eng. Sci. 17, 221, 1962.

27. Wise, D. L. and G. Houghton, Chem. Eng. Sci. 21, 999, 1966.

28. Wise, D. L. and G. Houghton, Chem. Eng. Sci. 23, 1211, 1968.

29. Pfeiffer, W. F. and I. M. Kreiger, J. Appl. Chem. 78, 2516, 1974.

30. Brockwell, J. L., PhD. Dissertation, The University of Toledo, 1979.

31. Brockwell, J. L. and K. J. De Witt, AICHE Annual Meeting, San Francisco, California, 1979.

32. Ward, C. A., A. Barakrishnan and F. C. Hooper, J. Basic Eng., 695, December, 1970.

33. Tucker, A. S. and C. A. Ward, J. Appl. Phys. 46, $4801,1975$.

34. Ward, C. A. and A. S. Tucker, J. Appl. Phys. 46, $233,1975$.

35. Ng, H. J. and D. B. Robinson, J. Chem. Eng. Data $\underline{23}, 325,1978$.

36. Yung, C. N., K. J. De Witt, J. L. Brockwell, J. McQuillen and A. Chai, Submitted to J. Colloid \& Interface Science, November, 1987. 


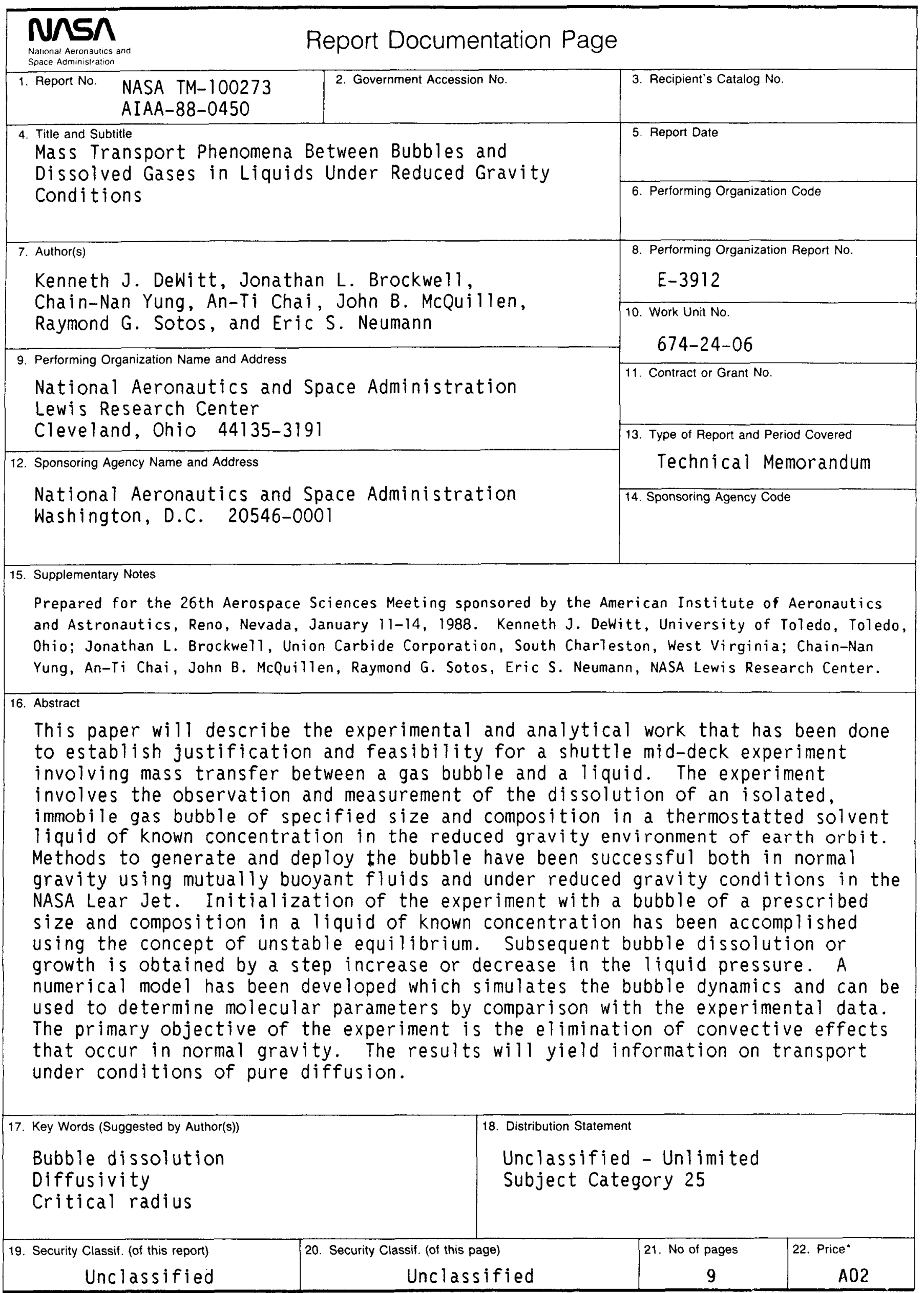

